\title{
Consultation on UTUC Stockholm 2018
}

\author{
Marianne Brehmer ${ }^{1} \cdot$ Palle Osther ${ }^{2}$
}

Published online: 7 September 2019

(c) Springer-Verlag GmbH Germany, part of Springer Nature 2019

Upper tract urothelial carcinoma (UTUC) is a rare but lethal disease. About $60 \%$ of tumours are invasive at the time of diagnosis. Radical nephroureterectomy (RNU) has been the standard treatment of UTUC. However, a number of studies have indicated that kidney-sparing surgery (KSS) and treatment with radical nephroureterectomy (RNU) offer equivalent long-term CSS if the right patients are selected [1,2].

The current guidelines outlined by the European Association of Urology (EAU) divide UTUC into low- and highrisk diseases [3] and recommend that kidney-sparing treatment should be discussed in all cases of low-risk tumour. However, due to the scarcity of the disease and that most published studies are retrospective with small number of patients included, there is relatively low level of evidence of the statements.

Studies have indicated stage and grade as strong prognostic factors $[4,5]$, whereas the impact of tumour size has been questioned [6]. Cytology and the small biopsies generally do not allow direct staging, but there seems to be a correlation between grade and stage [4], allowing indirect staging to some degree. However, correct grading has proven to be challenging [7-9].

All efforts to improve diagnostics are of importance. There is a need to identify possible better methods of grading, or to identify other more reliable diagnostic tools. Tumour heterogeneity may hinder correct grading from endoscopic biopsies. A better understanding of the genomics involved in UTUC is a highly interesting and advancing field [10], which may reveal more reliable prognostic markers.

Palle Osther

palle.joern.osther@rsyd.dk

Marianne Brehmer

mail@mbrehmer.com

1 Division of Urology, Department of Clinical Sciences, Danderyd Hospital, Karolinska Institutet, Stockholm, Sweden

2 Department of Urology, Urological Research Center, Lillebælt Hospital, University of Southern Denmark, Vejle, Denmark
Like low-risk UTUC seems to benefit from kidney-sparing treatment whereas the clear recommendation for organ confined high-risk UTUC is radical neprhoureterectomy with complete bladder-cuff removal. However, how different treatments should be carried out is a matter of debate. Furthermore, the role of lymph node dissection and the impact of decreased renal function after radical surgery are still issues needing to be clarified, as well as the role of diagnostic ureterorenoscopy for intravesical recurrence-just to mention a few aspects of controversies.

For a deeper discussion, to highlight difficulties in the diagnostic and therapeutic procedures in UTUC, we invited 20 experts in Europe to participate in the "Consultation on UTUC 2018. Aspects on diagnosis, treatment and follow-up, long-term results and risk stratification". The meeting took place at the Nobel Forum in Stockholm, September 2018.

The experts were assigned different topics within the field. They prepared presentations according to the standards for a scoping review by scanning the literature using Pubmed, Embase, and Web of Science. The meeting extended over 2 days, where the first day was a closed meeting for the experts, where all presentations were presented, discussed, challenged, and adjusted accordingly. The second day was an open meeting, where the adjusted presentations were presented and discussed to an audience with participants from all over Europe.

Conclusions drawn from the meeting were that when treating patients with UTUC, both tumour variables and patient's variables have to be taken into consideration. There is both a need and a possibility for personalized treatment decisions, which makes risk stratification necessary. However, for proper risk stratification prospective multicenter studies investigating and identifying better prognostic factors and optimizing diagnostic tools are needed, as well as strict long-term protocols for follow-up, in order to evaluate longterm results of different treatment modalities. It is our aim and hope that the conclusions from the presentations and the discussions will add to a deeper understanding of UTUC and inspire future research within the field. 
The meeting has resulted in three review papers [11-13] covering diagnosis of UTUC, treatment alternatives in UTUC and follow-up, long-term results and risk stratification in UTUC. The papers are, together with five original papers [14-18], published in this issue number on the topic.

\section{References}

1. Grasso M, Fishman AI, Cohen J, Alexander B (2012) Ureteroscopic and extirpative treatment of upper urinary tract urothelial carcinoma: a 15-year comprehensive review of 160 consecutive patients. BJU Int 110(11):1618-1626

2. Cutress ML, Stewart GD, Zakikhani P, Phipps S, Thomas BG, Tolley DA (2012) Ureteroscopic and percutaneous management of upper tract urothelial carcinoma (UTUC): systematic review. BJU Int 110(5):614-628

3. Rouprêt M, Babjuk M, Compérat E, Zigeuner R, Sylvester RJ, Burger $\mathrm{M}$ et al (2018) European association of urology guidelines on upper urinary tract urothelial carcinoma: 2017 update. Eur Urol 73(1):111-122

4. Holmäng S, Johansson SL (2005) Urothelial carcinoma of the upper urinary tract: comparison between the WHO/ISUP 1998 consensus classification and WHO 1999 classification system. Urology 66(2):274-278

5. Elawdy MM, Taha DE, Elbaset MA, Abouelkheir RT, Osman Y (2016) Histopathologic characteristics of upper tract urothelial carcinoma with an emphasis on their effect on cancer survival: a single-institute experience with 305 patients with long-term follow-up. Clin Genitourin Cancer 14(6):e609-e615

6. Villa L, Haddad M, Capitanio U, Somani BK, Cloutier J, Doizi $S$ et al (2018) Which patients with upper tract urothelial carcinoma can be safely treated with flexible ureteroscopy with holmium:YAG laser photoablation? Long-term results from a high volume institution. J Urol 199(1):66-73

7. Smith AK, Stephenson AJ, Lane BR, Larson BT, Thomas AA, Gong MC et al (2011) Inadequacy of biopsy for diagnosis of upper tract urothelial carcinoma: implications for conservative management. Urology 78(1):82-86

8. Wang JK, Tollefson MK, Krambeck AE, Trost LW, Thompson RH (2012) High rate of pathologic upgrading at nephroureterectomy for upper tract urothelial carcinoma. Urology 79(3):615-619
9. Dev HS, Poo S, Armitage J, Wiseman O, Shah N, Al-Hayek S (2017) Investigating upper urinary tract urothelial carcinomas: a single-centre 10-year experience. World J Urol 35(1):131-138

10. Kammerer-Jacquet SF, Mathieu R, Peyronnet B, Rioux-Leclercq N, Bensalah K (2017) Genomics in upper tract urothelial carcinoma. Curr Opin Urol 27(1):35-40

11. Fojecki G, Magnusson A, Traxer O, Baard J, Osther PJS, Jaremko $\mathrm{G}$ et al (2019) Consultation on UTUC, stockholm 2018 aspects of diagnosis of upper tract urothelial carcinoma. World J Urol. https ://doi.org/10.1007/s00345-019-02732-8

12. Jung H, Giusti G, Fajkovic H, Herrmann T, Jones R, Straub M et al (2019) Consultation on UTUC, stockholm 2018: aspects of treatment. World J Urol. https://doi.org/10.1007/s00345-01902811-w

13. Hasan MN, Rouprêt M, Keeley F, Cracco C, Jones R, Straub M et al (2019) Consultation on UTUC, stockholm 2018 aspects of risk stratification: long-term results and follow-up. World J Urol. https://doi.org/10.1007/s00345-019-02739-1

14. Malm C, Grahn A, Jaremko G, Tribukait B, Brehmer M (2019) Predicting invasiveness and disease-specific survival in upper tract urothelial carcinoma: identifying relevant clinical tumour characteristics. World J Urol. https://doi.org/10.1007/s0034 5-019-02760-4

15. Grahn A, Tanaka N, Uhlén P, Brehmer M (2019) Volumetric imaging: a potential tool to stage upper tract urothelial carcinoma. World J Urol. https://doi.org/10.1007/s00345-019-02682-1

16. Keller EX, Doizi S, Villa L, Traxer O (2019) Which flexible ureteroscope is the best for upper tract urothelial carcinoma treatment? World J Urol. https://doi.org/10.1007/s00345-019-02675-0

17. Campi R, Cotte J, Sessa F, Seisen T, Tellini R, Amparore D et al (2019) Robotic radical nephroureterectomy and segmental ureterectomy for upper tract urothelial carcinoma: a multi-institutional experience. World J Urol. https://doi.org/10.1007/s00345-01902790-y

18. Vartolomei MD, Iwata T, Roth B, Kimura S, Mathieu R, Ferro M et al (2019) Impact of alcohol consumption on the risk of developing bladder cancer: a systematic review and meta-analysis. World J Urol. https://doi.org/10.1007/s00345-019-02825-4

Publisher's Note Springer Nature remains neutral with regard to jurisdictional claims in published maps and institutional affiliations. 